\title{
The research on early warning of preventing the stampede on crowded places and evacuated technology
}

\author{
J. Wang ${ }^{1, a}$, Y. N. Ding ${ }^{2, b}$, D. D. LIU ${ }^{3, c^{*}}$ \\ ${ }^{1}$ School of Civil and Transportation Engineering, Beijing University of Civil Engineering and \\ Architecture, Beijing, China \\ ${ }^{2}$ School of Civil and Transportation Engineering, Beijing University of Civil Engineering and \\ Architecture, Beijing, China \\ ${ }^{3}$ School of Civil and Transportation Engineering, Beijing University of Civil Engineering and \\ Architecture, Beijing, China \\ a524632423@qq.com, ${ }^{\mathrm{b}}$ dextraordinary@126.com, ddongliu@sina.com
}

\begin{abstract}
Keywords: Dense crowds; Location of mobile phone; Safety precaution; Safety evacuation
Abstract: The conventional monitor mode of intensive crowd depends primarily on manpower or video surveillance, which employed to manage the crowd to prevent stampede is very difficult to implement effectively. For the purpose of judging the state aggregation of pedestrian in time, in this paper the correction coefficient is presented by comparing the measured with the mobile phone location data, based on mobile phone positioning technology to make the implementation of monitoring and alert classification of dense population more practical, in 2015 Beijing Ditan Temple Fair. Furthermore, through the numerical simulation analysis of evacuation of the BaiTai of Ditan' crowd, the reasons of delay time for evacuation have been found out as well as the advancement of supervision. This research method and the conclusion in this paper can be applied for early warning and forecast of preventing the stampede on crowded places and safety evacuation.
\end{abstract}

\section{Research Background}

Along with continuous economic and social development, acceleration of urbanization and gradual prosperity of commercial and trade activities, the risk of stampede accident resulting from dense crowds has been increasing, and the safety management of dense crowds has become more difficult. The conventional monitoring mode of intensive crowd depends primarily on manpower or video surveillance, which makes it very difficult to carry out real-time all-round monitoring in densely-populated areas with multimode vertical-crossing walking facilities and complicated internal distribution. Traditional on-site monitoring merely plays the role of alarming for disposal, and lacks the forecasting and early-warning of dense crowd risk, therefore making it difficult to attain the goal of "prevention beforehand". Therefore, it appears very necessary to set up a set of dense crowd risk monitoring and early-warning system by using the mobile phone location safety technology, judge the aggregation state of pedestrians in time, and control the behaviors that may give rise to risks.

At present, the theory of mobile phone network location is relatively mature. By collecting mobile phone network location data and connecting the path matching algorithm, we can acquire the travel tracks of mobile phone users, including travel time, average speed and travel distance information. By making full use of Internet, database and GIS technology, we can collect and analyze various key protected targets, danger sources and emergency data of cities, timely and effectively assemble various resources, carry out emergency measures, and offer assisted decision-making to emergency command, so as to reduce the threats of emergencies to resident health, property and life safety, perfect the emergency reaction mechanism of government to public emergency events, and set up an all-round "safety network" of emergency early-warning and treatment for cities.

The collection technology of traffic information based on mobile phone location has aroused the universal attention of many domestic and overseas scientific research institutes and technical companies, and has gradually become the hotspot frontier of domestic and overseas research. The 
overseas mobile phone location technology has developed to its maturity, and mobile phone location technology was applied to study the collection of traffic information in foreign countries at an earlier time. For example, INRETS took the lead in conducting the simulated experiment research of traffic information collection technology based on mobile phone location in 2000[1]. At present, the domestic research resources applied to traffic information collection and based on mobile phone location technology are relatively scattered, laying particular emphasis on theoretical aspects, e.g. travel feature study[2] and disaster search and rescue[3].

Aimed at the practice of preventing crowd stampede accident from occurring at dense-crowd places in Beijing Ditan Temple Fair in 2015, this thesis carries out field investigation analysis to dense-crowd places through the relevant technology of mobile phone location at Beijing Ditan Temple Fair, conducts real-time data analysis and hierarchical forecasting and early-warning by correcting error of mobile phone location data according to crowd density actual measurement data, conducts safe evacuation numerical simulation analysis to special places, puts forward the suggestion of crowd evacuation, and works out a set of forecasting and early-warning mechanism of preventing stampede in densely-populated places.

\section{Crowd density early-warning based on mobile phone location technology}

\section{The correction of mobile phone location data}

For its own sake, the existing statistic technology of crowd quantity always brings about some deviation between statistic data and actual data in terms of video recognition, mobile phone location technology, etc. The actual size of visitor flow plays a very important role to the successful monitoring and forecasting of crowd density risk. This thesis corrects the visitor flow data of mobile phone location by investigating the visitor flow and visitor density in various periods of Ditan Temple Fair.

\section{Actual measurement of crowd density}

The actual measurement occurred on February 18, 2015 (the Eve of Chinese New Year), from Xitian Gate to Memorial Archway. The safety check gates of Ditan Park from Xitian Gate to Memorial Archway are divided into Section 1 and Section 2 to count the actual number of tourists in groups. The visitor flow is counted once every $15 \mathrm{~min}$. Please refer to Fig 2 for the visitor statistics of actual measurement and mobile phone location.

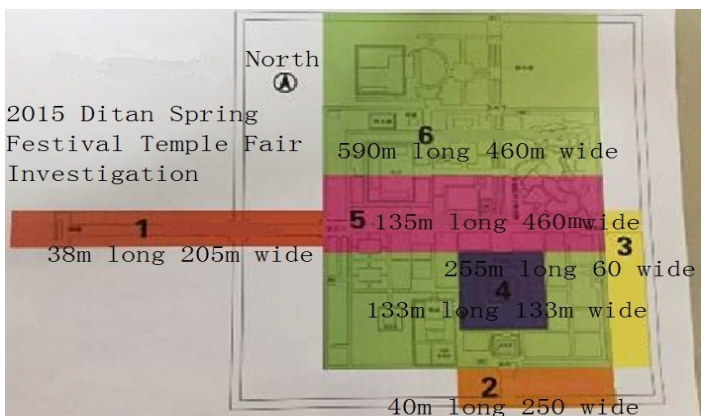

Figure 1. Ditan Spring Festival Temple Fair Investigation

\section{Analysis of actual measurement data and mobile phone location data}

At present, the mobile phone location user data provided by Mobile Company is that the user density in an area per $15 \mathrm{~min}$ is Pm. The site actual-measurement crowd density Pu is the actual user density of three telecom operators, and includes the error of mobile phone location technology. The ratio between the number of mobile phones per $15 \mathrm{~min}$ of Mobile Company and the actually-measured crowd density is $f=\mathrm{Pm} . / \mathrm{Pu}=0.195 / 0.14=1.39$ 


\section{Early-warning Mechanism}

\section{The significance of early-warning mechanism}

During each major holiday or festival, dense crowds often occur in such public places as park. Early-warning is making temporary judgments by experience in most cases. With regard to the question of "to what degree should the crowd density reach to make early-warning necessary?" Lu Chunxia, post-doctorate of Fudan University, proposed that the early-warning limit should be $0.75 \mathrm{~m}^{2} /$ person through due research. Mehdi Mesaad, scholar of Paul Sabatier University, and his colleagues, proved through experiment that individuals would be unable to control themselves due to lack of willpower when interpersonal distance is very small. Therefore, we need to classify the

Table 2: Visitor Density Precaution Level Classification

\begin{tabular}{|c|c|c|c|c|}
\hline Level & $\begin{array}{c}\text { Measured } \\
\text { visitor } \\
\text { density } \\
\text { Dp } \\
{\left[\mathrm{p} / \mathrm{m}^{2}\right]}\end{array}$ & $\begin{array}{l}\text { Mobile } \\
\text { phone } \\
\text { location } \\
\text { density } \\
\text { Dm } \\
{\left[\mathrm{p} / \mathrm{m}^{2}\right]}\end{array}$ & $\begin{array}{l}\text { Mobile } \\
\text { phone } \\
\text { location } \\
\text { visitors in } \\
\text { Area } 5\end{array}$ & Description \\
\hline 1 & $\geq 1.0$ & $\geq 1.39$ & $\geq 6516$ & $\mathrm{EC}$ \\
\hline 2 & {$[0.75,1.0)$} & {$[1.04,1.39)$} & {$[4876,6516)$} & $\mathrm{VC}$ \\
\hline 3 & {$[0.6,0.75)$} & {$[0.83,1.04)$} & {$[3891,4876)$} & $\mathrm{CC}$ \\
\hline 4 & {$[0.4,0.6)$} & {$[0.556,0.83)$} & {$[2625,3891)$} & $\mathrm{OC}$ \\
\hline
\end{tabular}
visitor density degrees, and conduct hierarchical precaution, and determine where the weak points are in the whole place according to early-warning hierarchies, and how to carry out rescue after any accident occurs, which appear very important.

\section{Classification of early-warning levels}

At present, there are no explicit standards on the classification of crowd density early-warning levels. The thermodynamic chart of Baidu is a product which superimposes different color blocks on the map to make a real-time description of crowd distribution, density and change trend, and a type of travel convenience service based on the big data of Baidu. Please refer to Table 1 for the classification of early-warning levels. The Interim Measures of Beijing on the Information Release Management of the Emergency Early-warning [4] classify early-warning information levels into Level 1, Level 2, Level 3 and Level 4 according to the extent of injury, the degree of urgency and the development trend, marked in red, orange, yellow and blue respectively. Level 1 is the highest level. In Table 2, Area 5 is 4688 square meters. The number of mobile location users in Area $5=$ the size of Area $5 \times$ the location density of mobile phone. Refer to Table 2 for the classification of visitor density early-warning levels.

\section{Forecasting and early-warning of crowd density of Ditan}

Refer to Table 3 for the real-time partial mobile phone location density dated February 18, 2015, and fig 3 and fig 4 are data summary figure. As can be learned from fig 4 , the largest density of mobile phone location in Area 1 on February 18, 2015 is 0.215 , indicating this the visitor flow in this area is in a normal state. The mobile phone location of Area 5 on February 18th began from 11:00, and the visitor flow of Area 5 had already reached a state of ordinary congestion, and Level-4 blue precaution. Ditan Temple Fair lasted from February 18 (Chinese New Year Eve) to February 25 (the 7th day of the first month according to Chinese calendar). From 9:00 am to 5:00 pm, the daily visitor flow was in a dense state. From 10:00 am to 2:00 pm was the peak of visitor flow.

According to the early-warning level set up in Table 2, we can learn from fig 3 and fig 4 the
Table 3 Mobile phone location density of Area 5 on February 19, 2015

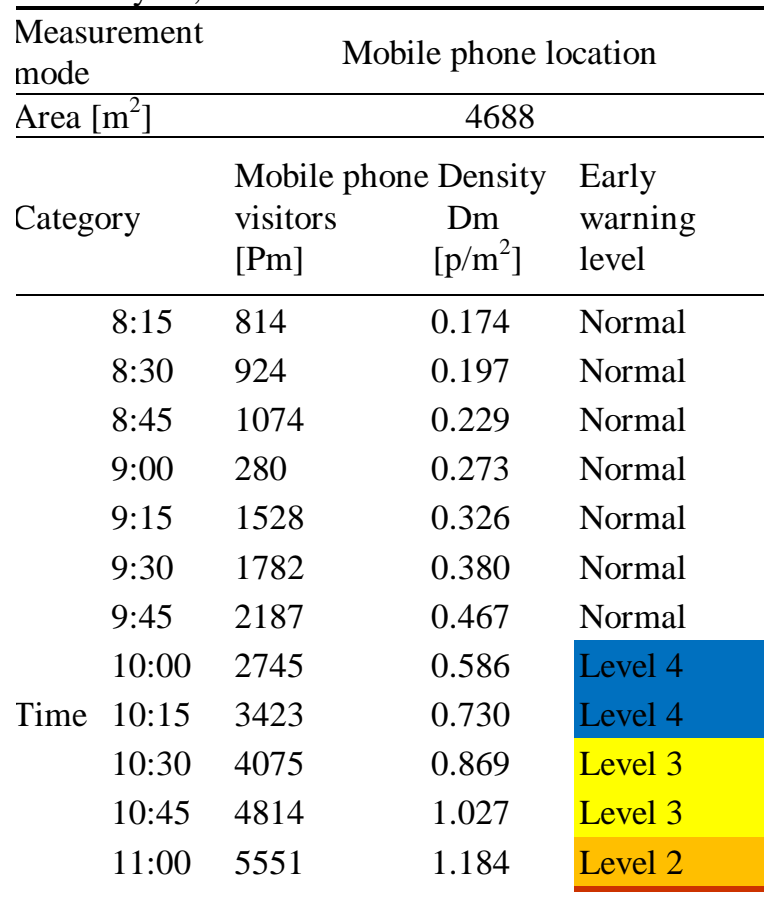


crowd quantity and density of Area 1 and Area 5.

From figure 4 we can see, on February 21, 2015, from 10:15 to 10: 30, the visitor flow of Area 5 reached the state of ordinary congestion and Level-4 blue precaution. At 10: 45, the visitor flow of Area 5 reached the state of considerable congestion and Level-3 yellow precaution. From 11:00 to 11:30, the visitor flow of Area 5 reached the state of high congestion and Level- 2 orange precaution. From 11:45 to 14:45, the visitor flow of Area 5 reached the state of extreme congestion and Level-1 red precaution. From 15:00 to 16:00, the visitor flow of Area 5 reached the state of high congestion and Level-2 orange precaution. At 16:45, the visitor flow of Area 5 reached the state of considerable congestion and Level-3 yellow precaution. At 16:30, the visitor flow of Area 5 reached the state of ordinary congestion and Level-4 blue precaution. Corresponding measures could be adopted according to the relevant early-warning situation. The largest density of mobile phone location in Area 5 on February 21 was Dm 1.623, far smaller than the largest density of mobile phone location in Area 5 on February 19 (Dm 2.779).

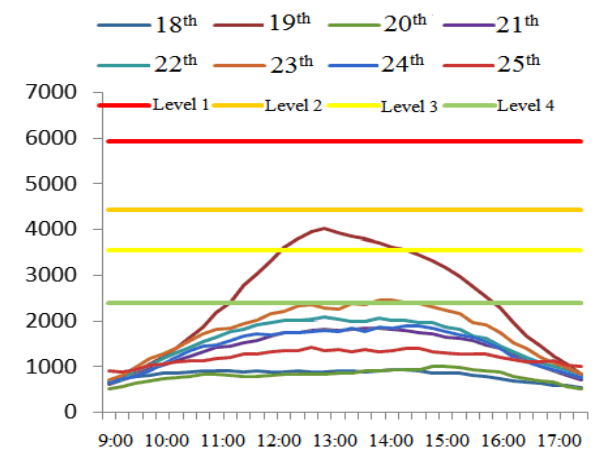

Figure.3 The Change Curve of Mobile Phone Orientation Visitor Number in Area 1

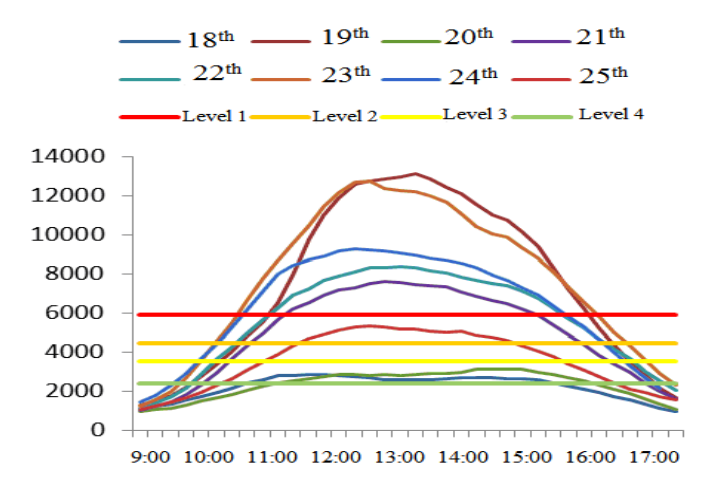

Figure.4 The Change Curve of Mobile Phone Orientation Visitor Number in Area 5

\section{Carrying out real-time monitoring by using mobile phone location}

At present, Mobile Company provides the original data of mobile phone users in the form of Excel document. The original data of users include the user flow in a certain period in a certain area, and cannot provide visitor density, and cannot indicate the early-warning information in a certain period according to the classified visitor density early-warning levels. Therefore, in order to promptly process the original data of mobile phone location users, graphically present the visitor flow and density in a certain period in a certain area and the current early-warning information, carry out the early-warning monitoring of crowd density, and timely and effectively provide emergency commanding with supporting decision-making, this thesis has developed the system of crowd gathering risk monitoring and early-warning information processing [5].

The main functions of this system include:

(1) Reading the original data of mobile phone location users. Use the way of document flow to read the original data documents of users, and deposit the read information in the memory;

(2) The pre-processing of original data of mobile phone location users. Use Poi control to conduct pre-processing to original data, including the processing of data format, redundant data information, etc.;

(3) Calculate the visitor density in different areas and in different periods. Calculate visitor density according to area size, time information, etc.;

(4) Divide the early-warning levels in different areas in different periods. According to the classification of visitor density levels, respectively calculate the early-warning information in different areas in different periods, including: red precaution, orange precaution, yellow early-warning and blue precaution.

(5) Calculate the largest visitor density in different areas. According to area information and visitor 
density information in different periods, calculate the current largest visitor density.

(6) Generating the monitoring and early-warning information report. Generate the monitoring and early-warning information forms, set up the fonts, frames, background, numeric type and other information, and finally set up a monitoring and early-warning information report.

The steps of system initiation and operation are:

(1) Initiate command prompt.

(2) Enter the current catalogue of procedures;

(3) Operate the command Java Emergency filename regionFlag.

Therein:

Filename stands for the original data document of mobile phone location users to be processed; regionFlag stands for the mark of divided area; 1 stands for Area 1; 5 stands for Area 5.

(5)If the following content occurs during system operation, the operation is successful.

(6)In the current catalogue of procedures, generate the monitoring and early-warning information report document output.xls.

Please refer to Appendix 1 for system source procedures.

\section{Baitai Evacuation Study}

Beijing Ditan Park is the largest earth sacrifice altar in China. A temple fair is held from the 30th day of the 12th month to the 7th day of the first month according to Chinese calendar, lasting for 8 days. During the temple fair, a sacrifice performance is held on the Workshop Platform from 10:00 to 10:30 am every day, marked by a large-scale occasion and a high visitor density. In order to avoid visitor congestion and stampede accident and other safety loopholes, we need to conduct safety evacuation research into densely-populated places. [6,7]

\section{The basic parameters of Baitai evacuation model}

According to the CAD data provided by Ditan Park, this thesis has set up an evacuation model of Baitai. As a two-storey building, Baitai covers an area of $17610 \mathrm{~m}^{2}$, including nine different areas (fig 5). We have set up the parameters for visitor density. Since Area 9 is for sacrifice performance, it is not involved in the change of simulated visitor density. Please refer to Table 4 for the width and number of the evacuation gate of Baitai and Table 5 for the parameters of visitor evacuation.

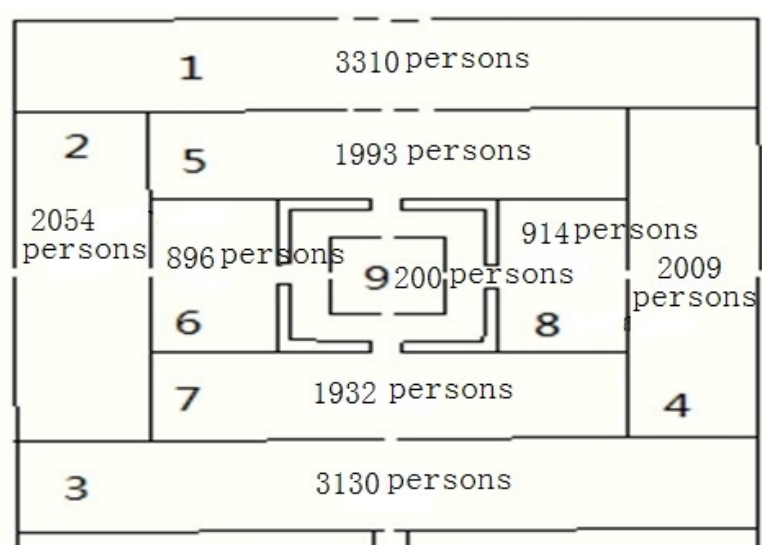

Figure.5 Ditan Baitai Evacuation Zoning Figure
Table 4 Baitai Basic Data

\begin{tabular}{lcc}
\hline & $\begin{array}{c}\text { Gate serial } \\
\text { number }\end{array}$ & Gate width \\
\cline { 3 - 3 } & & {$[\mathrm{m}]$} \\
\hline North gate & & \\
(west to east) & 2 & 3.43 \\
& 3 & 2.96 \\
& & 2.77 \\
West gate & 4 & 3.04 \\
East gate & 5 & 2.39 \\
South gate & 6 & 2.59 \\
\hline
\end{tabular}

Table 5: LEGION Evacuation Simulation Parameters

\begin{tabular}{ccccc}
\hline Area & Visitors & $\begin{array}{c}\text { Familiar } \\
\text { with the } \\
\text { site or not? }\end{array}$ & $\begin{array}{c}\text { Visitor } \\
\text { density }\end{array}$ & Evacuation \\
direction
\end{tabular}




\section{Baitai Evacuation Analysis}

During evacuation analysis, the evacuated crowd will be evacuated to the nearest exit. Since there are different distances among the gates facing east, south, west and north respectively, and each gate bears a different number of evacuated visitors, the evacuation time is different and the evacuation is delayed. The optimal evacuation scheme is that the time for completing evacuation of each gate is consistent.

In order to seek an optimized evacuation scheme, during evacuation calculation analysis, we have set up an iron fence between the east/west gate and the north gate (fig 6), and then move the iron fence, respectively calculate the evacuation time, and can finally find out the best location of iron fence, with the shortest evacuation time. This is exactly the location of iron fence or the work location of evacuator. Here, the evacuator guides the direction of visitor evacuation and shortens the evacuation time.

The visitor density of Evacuation Model 1 is $1 \mathrm{p} / \mathrm{m} 2$, and the evacuation time is 12 minutes and 34 seconds, and no iron fence is set up. Therein, the south gate and the east gate have the slowest

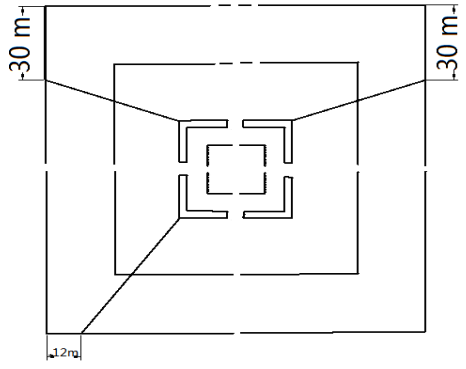

Figure.6 The location of iron fence evacuation, delaying the overall evacuation for 1 minute and 14 seconds. After many rounds of evacuation simulation optimization, Model 2 sets up iron fences 12 meters east of the southwest corner of Baitai and 30 meters south of the northeast and northwest corner of Baitai (fig 6). The evacuation time is 11 minutes and 52 seconds, 42 seconds (5.6\%) less than Model 1. fig 7 and fig 8 are respectively the visitor density thermal distribution diagrams of Model 1 and Model 2 when evacuated to the 10th minute. From the diagrams, we can obviously notice that the visitor density of the east, west and south of Model 2 is less and evener, with its evacuation time superior to that of Model 1.

The visitor density of Evacuation Model 3 is $2 \mathrm{p} / \mathrm{m} 2$, with an evacuation time of 23 minutes and 18 seconds (fig 9). From this, we can notice that the evacuation time obviously increases along with the rise of visitor density. When the visitor density increases one fold, the evacuation time will increase nearly $90 \%$. Therefore, we should regard controlling the visitor density of Baitai as one of important safety measures. The calculation analysis of evacuation indicates that we can adopt measures to increase the number of evacuees at the north gate to try to make the evacuation time of each gate consistent and shorten the evacuation time. After setting up iron fences, Evacuation Model 3 shortens evacuation time by 67 seconds and raises the evacuation speed by $4.6 \%$. According to the tactics of using the iron fences as evacuation boundaries, we can not only evacuate visitors, but also raise the evacuation efficiency.

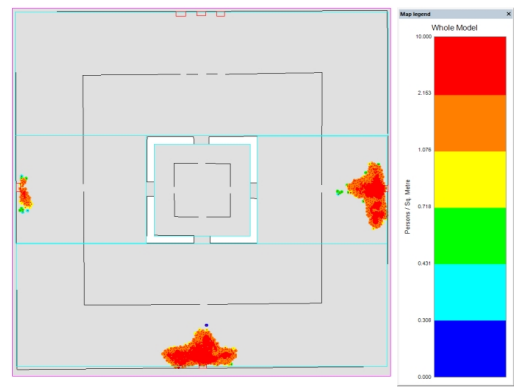

Figure.7Visitor Density Thermal Distribution Diagram in the 10th Minute of Model 1

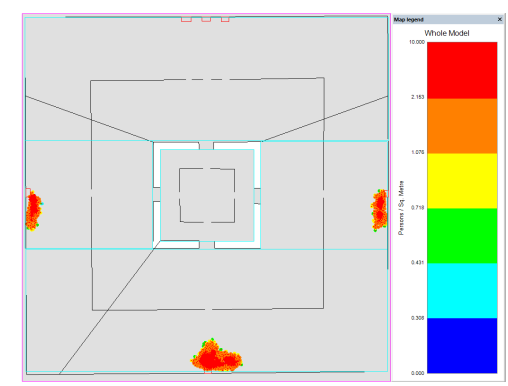

Figure.8Visitor Density Thermal Distribution Diagram in the 10th Minute of Model 2

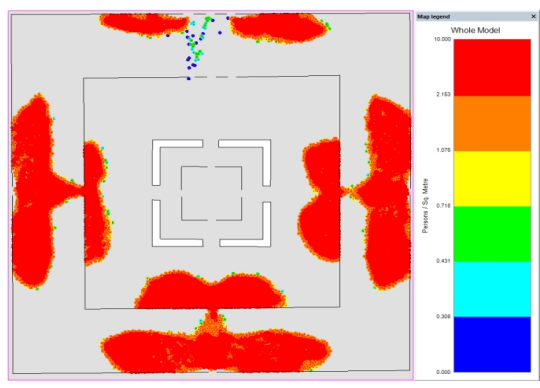

Figure. 9 Visitor Density Thermal Distribution Diagram in the 10th Minute of Model 3

\section{The evacuation suggestion measures of Baitai}

Through the numeric simulation of evacuation (visitor density is $1 \mathrm{p} / \mathrm{m}^{2}$ and $2 \mathrm{p} / \mathrm{m}^{2}$ ), we find out that the quickest direction of evacuation is the north side of Baitai, and next come the south side, east side and west side. We should guide tourists to evacuate from the north gate direction with a high evacuation speed. We can set up iron fences at proper locations to enable more visitors to evacuate 
from north gate and evenly distribute the visitors at the four exits. We can build iron fences into sliding gates, which are opened at ordinary times not to prevent tourists from visiting, and which are closed during evacuation. Therefore, we can use iron fences to more effectively organize visitors for evacuation.

We can learn by applying Legion safety evacuation for simulated analysis, the evacuation process of Ditan Baitai is difficult and the evacuation is slow when visitors are dense. Therefore, we can adopt the following evacuation measures at Baitai:

(1) Preferably control the visitor density of Baitai within $2 \sim 3 \mathrm{p} / \mathrm{m}^{2}$;

(2) Set up iron fences or arrange evacuators, so that organized evacuation can be conducted conveniently;

(3) Properly reduce the merchants adjacent to the east and west gates of Baitai, and expand the evacuation channel;

(4) Conduct an emergency plan drill. Through the drill, we can further fulfill the emergency plan, raise the rescue efficiency, and enable staff to be skilled at evacuation guidance procedures.

\section{Research conclusion and prospect}

This thesis uses mobile phone location technology for the first time to conduct crowd density risk early-warning study to the spring festival temple fair of Beijing Ditan in 2015, organically combines mobile phone data with architectural disaster prevention, enhances the reliability of traditional actual test investigation with the help of network data techniques, and offers effective reference to the early-warning mechanism of preventing high-density crowd from stampede. We also conduct safety evacuation analysis for Baitai. Research achievements can be extensively applied in South Luogu Alley, Back Lake and other landmark scenic spots in Beijing, large business circles and other areas. The research conclusion of this thesis is as follows:

(1) Set up the hierarchical real-time forecasting and early-warning mechanism based on mobile phone location, which is operated desirably at Beijing Ditan Temple Fair in 2015;

(2) Acquire the corrective coefficient of mobile phone location data through actual test, capable of being used as reference for the forecasting and early-warning methods of mobile phone location;

(3) Find out the reasons for the delay of evacuation of Baitai and put forward the management measures for improving safety evacuation through safety evacuation analysis.

\section{Acknowledgement}

The author of this thesis expresses his sincere gratitude to Beijing 2015 Ditan Spring Festival Temple Fair Organization Committee for its support, and to Professor Liu Dongdong, Ma Xiaoxuan and Li Zhihong for their patient guidance to this thesis, and to the postgraduate student Zhou Jingjing for his enthusiastic assistance to this thesis.

\section{Reference}

[1] Ygnaee, J.L. , Remy, J.G. \& Bosseboeuf, J.L. et al. Travel time estimates on Phone corridor network using cellular Phones as Probes:Phasel. Technology assessment and preliminary results[R]. France: INRETS, 2000.

[2] Chen, C. , Research into the User Travel and Stagnation Point Identification System Based on Mobile Phone Orbit Data [R]. Master's Theses of Beijing University of Technology, 2014;

[3] He, S.Q. \& Wang, T. Exploration into Earthquake Disaster Damage Report and Search and Rescue Technology Based on Mobile Phone Location Service[J]. South China Earthquake: 2012.12, 67-74.

[4] Beijing Emergency Office. Beijing Interim Measures on the Release Management of Emergency Early-warning Information, Beijing: Beijing Emergency Office. 2013.3.1.

[5] Gong, Y.G. \& Chen, X. Java Foundation of the Programming Design [M]. Beijing: Tsinghua University Press. 2009.7. 
[6] Gan, Y.H. , Chen, X.L. , Song, C. \& Lin, S.K. People Flow Traffic Simulation of Large-Scale Event [J]. Traffic Information and Safety: 2012.1, 30-33.

[7] Li, S.Y, Zhou, X., \& Liu, D.D. Analysis Research into Visitor Safety Evacuation of Ancient Architectural Complexes [J]. Engineering Earthquake Resistance and Reinforcement Supplement, 2014, 84-90. 